\title{
Effect of the sapphire substrate on spectral emission features of LEDs based on InGaN/AIGaN/GaN heterostructures
}

\author{
D.I. Bletskan ${ }^{1}$, O.R. Lukyanchuk ${ }^{1}$, O.D. Bletskan ${ }^{2}$ \\ ${ }^{1}$ Uzhgorod National University, 54 Voloshina str., 88000 Uzhgorod, Ukraine \\ Phone: +38(0312)616635, e-mail: bletskan@iss.univ.uzhgorod.ua \\ ${ }^{2}$ Scientific-Production Firm "Technocrystal", 5-a Granitna str., 88007, Uzhoorod, Ukraine
}

\begin{abstract}
Luminescence spectra of blue and green LEDs based on $\operatorname{In}_{\mathrm{x}} \mathrm{Ga}_{1-\mathrm{x}} \mathrm{N} / \mathrm{Al}_{\mathrm{y}} \mathrm{Ga}_{1-\mathrm{y}} \mathrm{N} / \mathrm{GaN}$ heterostructures with many quantum wells in the current range 0.1 to $10 \mathrm{~mA}$ have been studied besides the main electroluminescence band. In the emission spectra of blue LEDs, the energy maximum position is shifted with changing the current $\left(h v_{\max }=2.62 \div 2.63 \mathrm{eV}\right)$. A weak doublet of R-lines 692.8 and $694.3 \mathrm{~nm}$ conditioned by the re-emission of single $\mathrm{Cr}^{3+}$ ions in a sapphire substrate has been fixed. The use of substrates from heavy doped ruby for making white LEDs based on nitride heterostructures has been proposed. The expediency of using blue and green LEDs as sources of photoluminescence excitation in the range of R- and Nlines of sapphire substrates and heavy doped ruby has been shown.
\end{abstract}

Keywords: light-emitting diode, heterostructure, quantum well, electroluminescence, photoluminescence, sapphire, ruby.

Paper received 20.05.03; accepted for publication 17.06.03.

\section{Introduction}

Solution of the urgent problem to produce efficient LED sources of light operating in a randomly chosen visible spectral range was hindered for a long time by the problem of blue LEDs. A rather obvious way of supplementing the efficient red range LEDs based on $\mathrm{Al}_{\mathrm{x}} \mathrm{Ga}_{\mathrm{x}} \mathrm{As} / \mathrm{GaAs}$ heterostructures with the devices based on wider-bandgap semiconductors $\mathrm{A}^{\mathrm{III}} \mathrm{B}^{\mathrm{V}}$ appeared to be rather long and requiring considerable efforts of physicists and technologists. Wider gap GaP, due to its non-direct band character, has been used only in the mode of impurity luminescence with a low efficiency in the green-yellow spectral range. The success of the industrial technology of nitride heterostructures on sapphire substrates became real only recently due to improvement of the method of gas-phase epitaxy from metal-organic compounds [1].

The use of structures with single and numerous quantum wells (QW), which ensure size quantization of the energy spectrum of carriers and their effective irradiative recombination proved to be significant. With the creation of LEDs based on InGaN/AlGaN/GaN heterostructures possessing quantum wells of several $\mathrm{nm}$, it became possible to overlap the whole blue-green range of the spectrum (450-540 nm) with the quantum efficiency reaching $\approx 10 \%$ and the light efficiency up to $60 \mathrm{~lm} / \mathrm{W}$ [1]. Such parameters are realized in industrial blue and green LEDs of the Nichia firm (Japan), which have been the object of this paper.

\section{Experimental}

Blue and green LEDs based on $\operatorname{In}_{\mathrm{x}} \mathrm{Ga}_{1-\mathrm{x}} \mathrm{N} / \mathrm{Al}_{\mathrm{y}} \mathrm{Ga}_{1-\mathrm{y}} \mathrm{N} / \mathrm{GaN}$ heterostructures grown by the gas-phase epitaxy methods from metal-organic compounds on sapphire substrates have been studied. In these structures, the active $\operatorname{In}_{x} \mathrm{Ga}_{1-\mathrm{x}} \mathrm{N}$ layer included many quantum wells. The wavelength in the emission maximum is set by the chemical composition of the active layer and for the blue-green range of the spectrum the value of $x$ varies within $0.2 \div 0.43$. 20 LEDs of each color have been investigated.

The emission spectra were investigated within the interval of currents $I=0.1 \div 40 \mathrm{~mA}$ using the DFS-24 complex.

\section{Results and discussion}

Electroluminescence spectra measured at room temperature for current values from the range $1 \div 40 \mathrm{~mA}$ (Fig. 1) 

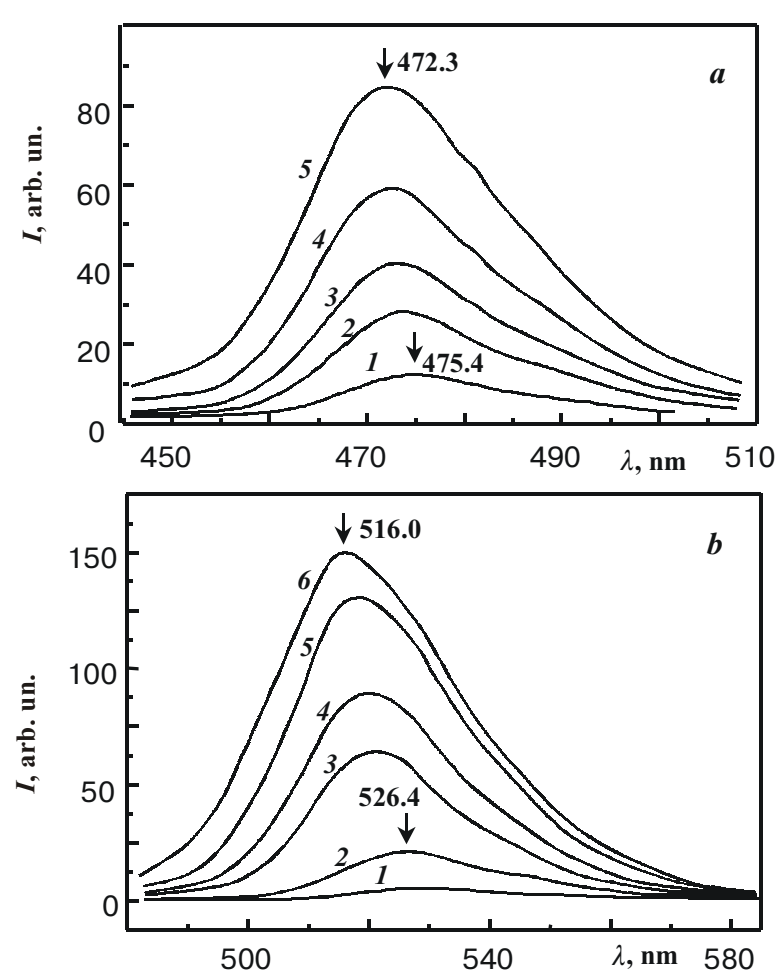

Fig. 1. Electroluminescence spectra of blue $(a)$ and green $(b)$ LEDs based on InGaN/AlGaN/GaN heterostructures with many quantum wells at different currents. I, mA: (a) $1-3,2-6,3-9$, $4-15,5-25$; (b) $1-1,2-3,3-6,4-9,5-25,6-40$.

correlate rather well with the data obtained for analogous structures in the recent publication [2]. We found also the revealed in [2] effect of the high-energy shift of the emission band maximum approximately by $15 \mathrm{meV}$ with the increase of current in the above mentioned range. In spite of its relatively large halfwidth, the emission band $\left(\Delta \lambda_{1 / 2} \approx 25 \mathrm{~nm}\right)$ is elementary. In the case of multi-well structure, we did not find the additional long wave emission maximum related to the interbarrier tunneling, which was found in [3] for the structures with single $\operatorname{In}_{x} \mathrm{Ga}_{1-\mathrm{x}} \mathrm{N}$ quantum well.
Fig. 2 presents in detail the long wave range of the emission spectrum of LED under consideration. It fixes a weak (in comparison with the main blue wide emission band) doublet of lines 692.8 and $694.3 \mathrm{~nm}$. These values coincide with the well-known photoluminescence (PL) Rlines of low-doped ruby, which are effectively excited in lasers by the xenon lamp irradiation. A relatively low intensity of R-lines in our case is determined by two factors. Firstly, when producing LEDs, the epitaxy of semiconductor layers was made on to perfect substrates cut from specially non-doped sapphire crystals [4]. That is why some background amount of chromium doping them is at least several orders lower than its amount in laser ruby. Secondly, the non-comparable inefficiency conditions of PL ion excitation by superintensive practically white light of xenon lamps, on the one hand, and the blue LED irradiation spectrum, on the other hand. Taking into account that there are two intensive wide bands $Y$ $\left(\lambda_{\max }=410 \mathrm{~nm}\right)$ and $\mathrm{U}\left(\lambda_{\max }=560 \mathrm{~nm}\right)$ and the lowintensive B line (a triplet of 476.3, 474.6, $468.3 \mathrm{~nm}$ ) in the absorption spectrum of $\alpha-\mathrm{Al}_{2} \mathrm{O}_{3}: \mathrm{Cr}^{3+}$ crystals, it becomes evident that excitation is realized practically only through weak absorption bands. It should be noted that for green LEDs based on InGaN/AlGaN/GaN heterostructures, the electroluminescence spectrum $\left(\lambda_{\max }=505\right.$ $520 \mathrm{~nm}$ ) is somewhat better overlapped by its longwave wing with the spectrum of chromium ion excitation using $\mathrm{U}$-band in $\alpha-\mathrm{Al}_{2} \mathrm{O}_{3}$. That is why green LEDs emission is more effective for photoluminescence excitation in Rlines.

To prove the above-mentioned considerations, we made additional comparative photoluminescence measurements of sapphire substrates [4] and concentrated ruby crystals, grown by the Verneil method. The PL spectra were compared providing excitation with blue and green LEDs as well as He-Ne laser of the power near $20 \mathrm{~mW}$.

The obtained results are presented in Fig. 3. It illustrates that at the equal level of luminescence, the excitation of R-lines for ruby is more intensive than in sapphire substrates. Besides, the comparison of the first and second curves proves more effective PL excitation with a
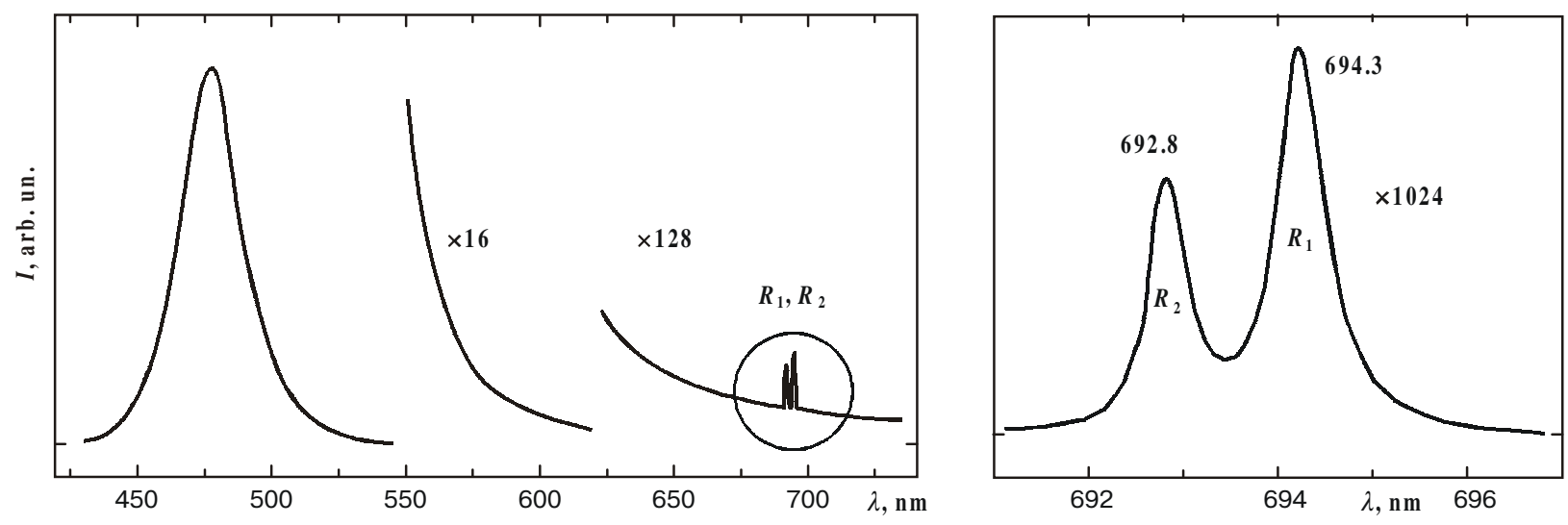

Fig. 2. Electroluminescence spectra of blue LED based on InGaN/A1GaN/GaN heterostructure. 


\section{D.I. Bletskan et al.: Effect of the sapphire substrate on spectral emission ...}
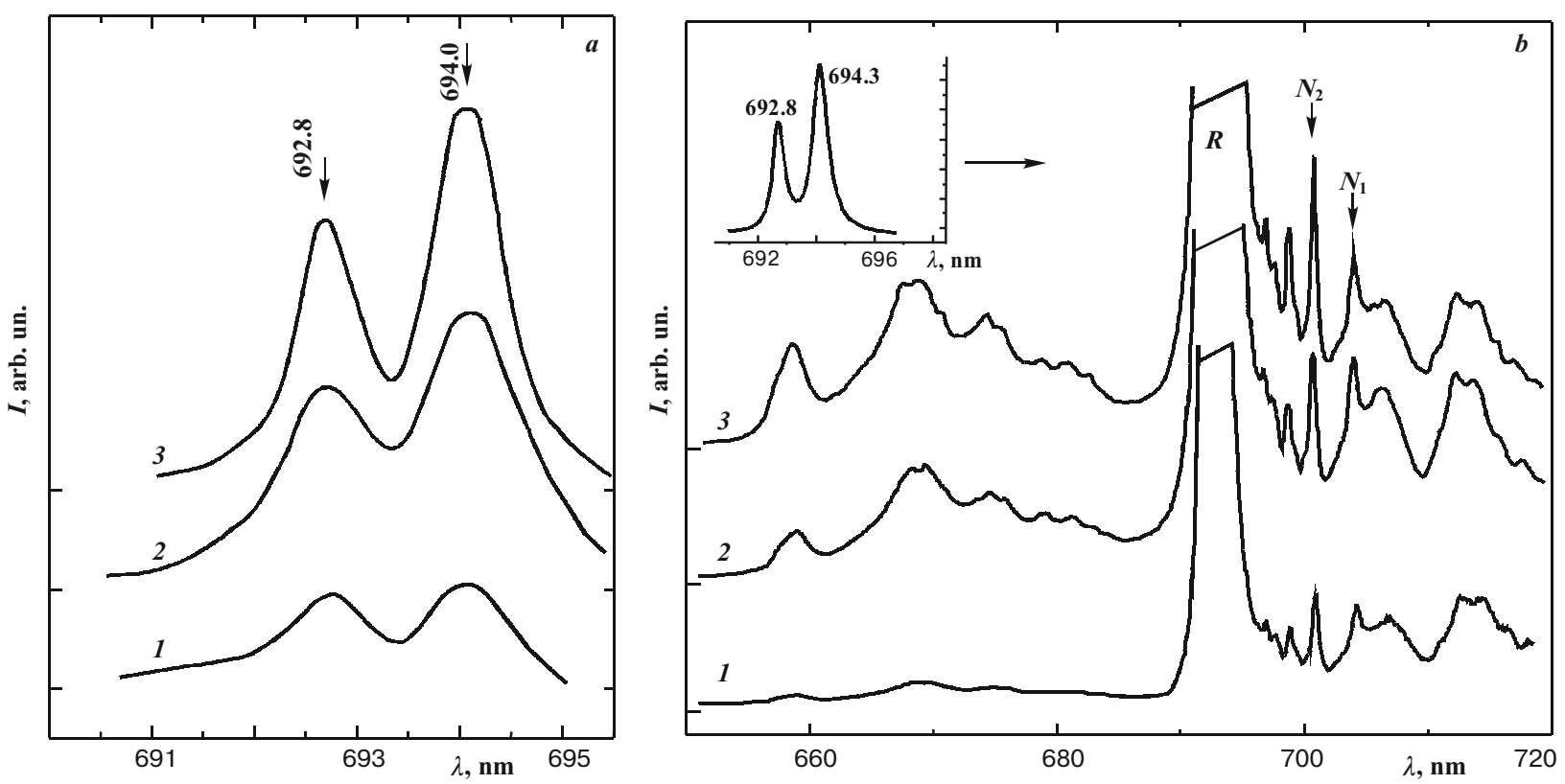

Fig. 3. Photoluminescence spectra of sapphire substrate $(a)$ and heavy doped ruby $(b)$ at excitation by blue (l) and green (2) LEDs and He-Ne laser $(\lambda=632.8 \mathrm{~nm})(3)$.

green LED in comparison with a blue one, as well as their approximately equal light efficiency.

To end with, we note an interesting possibility to practicaly use the discussed effect of red re-emission in sapphire substrates with applied to them InGaN/AlGaN/ $\mathrm{GaN}$ heterostructures, which irradiate in blue and green spectral. It is connected with the creation of LEDs with white luminescence. Currently they are realized on the basis of blue LEDs $\left(\lambda_{\max }=470 \mathrm{~nm}\right)$ by coating them with luminophore that emits in a broad spectral range overlapping the green-yellow and red parts. The total luminescence of the LED and the excited by it luminophore is perceived by human eyes as white.

It is important to discuss another possibility of producing white LEDs. By varying the componential composition and the thickness of quantum wells in InGaN/ $\mathrm{AlGaN} / \mathrm{GaN}$ multiwell structure it is possible to realize the structure which is a combination of blue and green LEDs, that emits simultaneously in both spectral ranges. If such a structure is grown using as a substrate a ruby plate $\left(\alpha-\mathrm{Al}_{2} \mathrm{O}_{3}\right.$ specially doped by $\left.\mathrm{Cr}^{3+}\right)$, it will provide an additional red irradiation from the substrate at the expense of not only the re-emission in R-lines, but also in a much broader red area including the irradiation in $\mathrm{N}$ - lines. In the result of the total effect, white luminescence may be obtained. The efficiency of the LED and the "purity" of white spectrum will be determined by the correlation of the intensities of blue and green constituents and their spectral coordination with Y- and U-absorption bands.

\section{References}

1. S. Nakamura, Introduction to nitride semiconductor blue lasers and light emitting diodes. // Taylor Francis. London. 2000.

2. V.E. Kudryashov, S.S. Mamakin, A.E. Yunovich, A.N Kovalov, F.I. Maniahin, Spectra and quantum output of irradiation of LEDs with quantum wells based on heterostructures from GaN - current and voltage dependency. // Fiz. Tekhn. Poluprov., 35(7), pp. 861-867 (2001), in Russian.

3. V.E. Kudryashov, K.G. Zolin, A.N. Turkin, A.E. Yunovich, A.N. Kovalov, F.I. Maniahin, Tunnel effects in LEDs based on InGaN/AlGaN/GaN heterostructures with quantum wells. // Fiz. Tekhn. Poluprov., 31(11), pp. 1304-1309 (1997), in Russian.

4. D.I. Bletskan, O.D. Bletskan, O.R. Lukyanchuk, A.I. Mashkov, J.M. Pekar, Mechanic sapphire crystals processing and producing substrates from them. // Naukovi visnik Uzghorodskogo Universitetu. Seria Fizika, 11, pp. 161-173 (2000), in Ukrainian. 